\title{
Polysèmes
}

Revue d'études intertextuelles et intermédiales

\section{Le sphinx noir et la baleine blanche : peinture de l'ineffable et chromatisme du silence dans Moby- Dick}

The Black Sphinx and the White Whale: a Picture of the Indescribable and the

Chromatics of Silence in Melville's Moby-Dick

\section{Lise Revol-Marzouk}

\section{OpenEdition}

Journals

Édition électronique

URL : http://journals.openedition.org/polysemes/486

DOI : $10.4000 /$ polysemes. 486

ISSN : 2496-4212

Éditeur

SAIT

\section{Référence électronique}

Lise Revol-Marzouk, « Le sphinx noir et la baleine blanche : peinture de l'ineffable et chromatisme du silence dans Moby-Dick», Polysèmes [En ligne], 14 | 2015, mis en ligne le 18 novembre 2015, consulté le 10 octobre 2020. URL : http://journals.openedition.org/polysemes/486 ; DOI : https://doi.org/ 10.4000 /polysemes.486

Ce document a été généré automatiquement le 10 octobre 2020

Polysèmes 


\title{
Le sphinx noir et la baleine blanche : peinture de l'ineffable et chromatisme du silence dans Moby- Dick
}

\author{
The Black Sphinx and the White Whale: a Picture of the Indescribable and the \\ Chromatics of Silence in Melville's Moby-Dick
}

Lise Revol-Marzouk

1 Au cœur de Moby-Dick le lecteur assiste à la capture, au dépeçage puis à la décapitation d'un gigantesque cachalot «brillant d'une couleur éthiopienne» (" of an Ethiopian hue », Melville 1851a, 308). Tandis que son corps pelé est rendu, tel un «tombeau de marbre blanc » («marble sepulchre », Melville 1851a, 336), à l'immensité marine, sa tête immense, encapuchonnée de noir, est hissée sur le flanc du Pequod. C'est là, au chapitre 70, que le capitaine Achab vient la retrouver pour l'interroger, dans une mise en scène aux accents tragiques, sur les secrets des abîmes. Intitulé "The Sphynx ", l'épisode mêle habilement des traits empruntés à la double origine culturelle de la figure mythique : la scénographie hellénique d'ensemble, souvenir de la confrontation entre Ædipe et la Sphin $x^{1}$ dans la légende thébaine, s'enrichit ainsi de signes résolument égyptisants, réminiscences du grand Sphinx de Gizeh ${ }^{2}$. La tentative s'avère vaine cependant. Enfermé dans un mutisme sépulcral, le crâne obscur se refuse à livrer ses mystères.

2 La vision mythique et le jeu chromatique qui l'accompagne ne sont assurément pas anodins. Dans ce roman conçu comme une œuvre close, où tout détail constitue un reflet de l'ensemble, tout incident une préfiguration du naufrage final, tout cétacé, un double de Moby-Dick, le sphinx noir est appréhendé en lieu et place de la baleine blanche. L'interrogation concrète et référentielle du cachalot-sphinx par Achab sur le pont du Pequod redouble ainsi naturellement son interrogation abstraite et littéraire par Ismaël sur la terre ferme. L'une et l'autre perspectives ne sont pas opposées, puisque le discours ismaëlien, comme de coutume, englobe et assume le discours 
d'Achab dans la totalité de l'approche narrative. C'est bien le narrateur en effet qui, reprenant les termes du capitaine, choisit de désigner la tête du cachalot du nom de la figure mythologique pour en faire le titre d'un chapitre. Au passé de l'aventure halieutique, poursuite matérielle de la baleine, se superpose le présent de l'aventure littéraire, poursuite textuelle de sa forme. Rets du langage et harpon de la nomination traquent eux aussi, inlassablement, une proie insaisissable. Pour celui qui, jeune homme, délaissa l'enseignement pour se faire matelot traversant l'Atlantique, avant de s'engager vers les mers du sud sur une baleinière, l'océan et ses créatures des profondeurs constituent assurément un miroir métaphorique de prédilection. C'est donc, au propre et au figuré, en abyme qu'il convient de considérer ce sphinx, hissé à la surface de la mer et du texte par Melville.

3 Esquisse mythologique tracée en lettres noires sur le fond blanc de la baleine et de la page, capture métaphorique par le réseau imaginaire du texte, le sphinx propose quelque chose comme une image enfin tangible de l'objet fuyant du discours ismaëlien. Reste à définir ce que cette « investiture mythique ${ }^{3}$ donne vraiment à voir de MobyDick. Qu'est-ce qui précisément de la baleine blanche accède par cette tête noire à la représentation ? Pour y répondre, il nous faut, à l'instar d'Ismaël, entreprendre à notre tour une circumnavigation de la tête décapitée et tenter d'en déchiffrer les lignes absconses. Ceci implique un certain goût de l'aventure mythocritique, avec son lot d'errances et d'incertitudes. Il convient ainsi d'en revenir à l'épreuve originelle de la décapitation, considérée dans sa double dimension cétologique et littéraire, pour interroger, jusque dans leurs contradictions, les déclarations programmatiques du narrateur et l'ambivalence chromatique qui en procède.

\section{Fonction picturale 1 : une « peinture » noire de la baleine blanche}

4 Au début du chapitre 55, Ismaël promet au lecteur une «peinture » de la forme vraie de la baleine, établissant d'emblée la dimension picturale de son entreprise littéraire :

I shall here long paint to you as well as one can without canvas, something like the true form of the whale as he actually appears to the eye of the whaleman when in his own absolute body the whale is moored alongside the whale-ship so that he can be fairly stepped upon there. (Melville 1851a, 285)

Substituer le tissu (textum) romanesque à la toile du tableau maritime, tel est bien le projet du narrateur. Les trente-deux chapitres suivants, qui constituent, selon l'expression d'Howard Vincent, le « centre cétologique » de l'œuvre (Vincent 121), sont tout entiers consacrés à la poursuite de cette ambition. Il est notable dans cette perspective que la figure noire du sphinx apparaisse, de manière quasi mathématique, au milieu exact de cette série de chapitres. À n'en pas douter en effet, le surgissement de la vision mythique au chapitre 70 , amarrée elle aussi sur le flanc du navire, participe directement de la peinture annoncée.

6 Voilà qui explique le caractère essentiellement visuel de la double référence mythique. Appuyé sur la bêche qui a servi à décapiter l'animal, disposée sous son bras comme une béquille, prêt à affronter oralement le monstre, Achab s'identifie en premier lieu à l'adversaire de la sphinx questionneuse des représentations helléniques ${ }^{4}$. Pour qui en douterait, la théâtralité de la mise en scène, définissant décor, accessoires et place du 
protagoniste, s'avère révélatrice. Sur les planches du Pequod, c'est bien un monologue tragique qui se prépare :

A short space elapsed, and up into this noiselessness came Ahab alone from his cabin. Taking a few turns on the quarter-deck, he paused to gaze over the side, then slowly getting into the main-chains he took Stubb's long spade-still remaining there after the whale's decapitation-and striking it into the lower part of the halfsuspended mass, placed its other end crutch-wise under one arm, and so stood leaning over with his eyes attentively fixed on this head. (Melville 1851a, 339)

7 Parallèlement se dessine, en filigrane, l'image du grand Sphinx de Gizeh. L'association procède ici plus particulièrement de l'identité formelle entre le crâne décapité du cachalot maintenu à la surface de l'eau et le chef gigantesque du Sphinx de Gizeh qui émerge à peine des sables en ce milieu de XIX ${ }^{\mathrm{e}}$ siècle ${ }^{5}$. Développés à l'envi, les deux axes paradigmatiques du silence et de l'immensité remotivent métaphoriquement et chromatiquement le topos analogique océan/désert: le pont du bateau, silencieux, est « désert» (« deserted deck») et le calme "cuivré » (« copper-calm»), "pareil à un universel lotus jaune » (« like a universal yellow lotus »), suggère la couleur des dunes. Le lecteur se trouve ainsi invité à puiser dans la mémoire collective de la campagne d'Égypte - planches, dessins et gravures rapportés du plateau de Gizeh par les savants, artistes et touristes - la cause première de la ressemblance. De cette identification initiale procède une série d'associations secondaires, permettant de filer, en imagination, la comparaison: tête encapuchonnée, évoquant un némès égyptien, absence remarquée de barbe, rappelant la perte de l'attribut pharaonique du grand Sphinx, soleil de midi, souvenir de la fonction solaire de la statue, outils de pêche et travail cétologique, mimétiques de la conquête archéologique. Avant qu'Achab ne prenne la parole, la référence se fait d'ailleurs explicite: «It was a black and hooded head; and hanging there in the midst of so intense a calm, it seemed the Sphynx's in the desert » (Melville 1851a, 339).

8 Avec son cortège d'associations imaginaires multicolores, le surgissement de la figure noire du sphinx répond bien donc, paradoxalement, à une exigence de figuration de la baleine blanche. Et ce d'autant plus que Moby-Dick est définie précisément, à plusieurs reprises, par son absence de face caractéristique : "And hint what he will about his face, I say again he has no face " (Melville 1851a, 414). L'épisode de la décapitation prend tout son sens dans cette perspective. En même temps qu'il permet d'esquisser, au cœur du roman, l'image compensatoire du sphinx, il rend en effet à la mer son corps dépecé, «immense et blanc fantôme sans tête flottant toujours plus loin du navire " ("The vast white headless phantom floats further and further from the ship », Melville 1851a, 336), réminiscence quasi explicite, tant par l'absence de couleur que par le mouvement, du corps fuyant de Moby-Dick, "majestueux fantôme encapuchonné, pareil à une colline enneigée » « flottant » dans l'âme du narrateur (« there floated into my inmost soul [...] one grand hooded phantom, like a snow hill in the air » Melville 1851a, 8).

9 Telle est donc la fonction figurative dévolue à la tête sphinxiale, forme noire séparée d'un corps blanc, informe et fuyant. Il s'agit bien, au moyen d'une émergence imagée, de substituer à l'inaccessibilité du corpus absent de l'œuvre l'alternative d'une figure identifiable, silhouette obscure accompagnée d'analogies visuelles polychromes. Image différentielle empruntée à un ailleurs référentiel, ébauche de picturalité, tête sortie de l'imaginaire collectif pour marquer, stylet victorieux, la page de l'insaisissable cétologique de la noirceur du signe métaphorique. 


\section{Fonction picturale 2 : noirceur et blankness, une peinture « en négatif »}

10 Le lecteur averti ne saurait pourtant ignorer le double discours du narrateur qui condamne, en même temps qu'il s'y adonne, toute entreprise de cette envergure. MobyDick lui apparaît en effet dès le chapitre 55, en contradiction totale avec ses déclarations d'intention, comme la seule créature à devoir échapper à toute peinture :

For all these reasons, then, any way you may look at it, you must needs conclude that the great Leviathan is that one creature in the world which must remain unpainted to the last. (Melville 1851a, 289)

11 L'exploration des portraits passés de la bête le conduit ainsi à souligner le caractère approximatif, voire erroné, des figurations mythologiques, notamment égyptiennes et grecques, des dessins de voyage et des images scientifiques. On ne saurait saper avec plus d'ironie la représentation sphinxiale. Et ce d'autant plus que l'échec de ces tentatives tient précisément à leur volonté de s'effectuer à partir d'un modèle extrait des profondeurs marines, hissé à l'air libre, privé de ses ondulations naturelles, figé enfin dans son portrait comme dans la mort. Il y a là, en écho, la stigmatisation explicite de l'esquisse tracée au chapitre 70. Il y a également, en filigrane, la dénonciation de son complémentaire, à savoir le dépeçage du corps décapité. Tombeau de marbre, fantôme sans tête soumis, à la surface des flots, aux vautours de la mer, continuellement profané, le corps blanc du cachalot-sphinx n'est qu'un double sépulcral et superficiel de la très vivante baleine qui se meut dans les abîmes. Et si, comme l'affirme le narrateur, il y a la même relation du squelette mort du léviathan à l'animal vivant que du papillon à sa chrysalide ${ }^{6}$, alors c'est bien à un insecte mort, épinglé sur la page blanche du texte, que doit s'apparenter le chef noir du cachalot en sa comparaison sphinxiale. Crâne noir, nu, rigide et muet, grande figure crépusculaire traversée du stylet métaphorique, le cachalot-sphinx décapité ne serait plus qu'un de ces vulgaires papillons de nuit nommés "sphinx tête de mort $\rrbracket^{7}$, cloué au musée du texte, vieille galerie d'histoire naturelle aux portraits mythologiques erronés.

À relire dans cette perspective le chapitre 70 , on ne peut qu'être frappé par la fondamentale négation qui caractérise l'émergence mythologique. Quoique doté d'une existence iconographique faisant appel à l'imaginaire du lecteur, le visage esquissé ne laisse pas en effet, dans les mots, d'être traversé de non-être. Il semble qu'accède ainsi à la représentation non pas tant la face cachée de Moby-Dick que l'impossibilité même de sa représentation.

13 C'est, en premier lieu, sous le signe de l'absence que se trace l'obscur portrait sphinxial. Du chef décapité du cachalot-sphinx, on apprend seulement en effet qu'il est noir, encapuchonné et dépourvu de barbe. Rien de plus. Pas d'évocation du regard, des oreilles, du nez; pas d'indication précise de forme ni de taille; une vague immensité gage d'indétermination, et, çà et là, quelques mousses, blanches comme il se doit. Tout le reste, à savoir le cadre égyptisant polychrome et la scénographie hellénique que nous avons relevés, n'est que reconstruction métaphorique substituée à la représentation véritable du monument, concrétion de poncifs contextuels visant à masquer le défaut de visibilité de la statue, images d'Epinal archéologiques et légendaires couvrant l'absence du monstre. Tout le reste ne fait ainsi qu'accentuer, en le soulignant, le défaut même de représentation sphinxiale alors à l'œuvre. 

sphinx, privée de tout trait distinctif explicite, n'en participe ainsi pas moins, elle aussi, de ces silhouettes indécises, monstres blancs, que sont l'ours polaire, le requin blanc, l'Albatros, le squid et tous les autres doubles de Moby-Dick. Figure en creux, figure vide, vague trace mnémonique, que cette tête métaphorique sans véritable figure. Tout à son désert descriptif, elle ne propose pas, en définitive, une vision plus juste de l'animal que les dessins des peintres anglais et américains, qui s'en tiennent, nous dit Ismaël, à « un contour schématique » (« mechanical outline »), traçant « un profil vide de la baleine » («vacant profile») identifié précisément par le narrateur, s'en étonnera-t-on, à un «profil de pyramide» («the profile of a pyramid», Melville 1851a, 292), autre monument sépulcral égyptien. sphinxiale, simple profil d'ombre, loin de remettre en cause la blancheur des choses et des signes, ne fait-elle qu'en exhiber, pour ainsi dire en négatif, leur essentielle insaisissabilité. Si la tête de sphinx, en sa noirceur, montre quelque chose en effet, c'est assurément ce point extrême, évoqué à la fin du célèbre chapitre 42 sur la blancheur, que laisse présager la surdétermination du blanc: la vacuité, ou plutôt, en un terme tiré, étymologiquement, vers la blancheur, la blankness. Et le sphinx noir de s'ériger, à l'instar de la blancheur elle-même, en une figuration de l'anéantissement, véritable représentation du vide, « absence visible » (« visible absence »).

\section{Fonction synesthésique : le sphinx noir, la blancheur et le silence}

16 Cet imaginaire s'accompagne, dans le cas de la couleur blanche comme dans celui du sphinx noir, d'un déplacement synesthésique. C'est que l'invisible se double, à la lumière de la symbolique sphinxiale, de l'indicible. La question n'est plus tant dès lors de montrer ce qui se soustrait à la représentation sous les traits du sphinx que de le figurer au travers de son silence. Déplacement remarquable de la vision à la parole que rend particulièrement sensible l'adresse d'Achab au monstre :

"Speak, thou vast and venerable head", muttered Ahab, "which, though ungarnished with a beard, yet here and there lookest hoary with mosses; speak, mighty head, and tell us the secret that is in thee. Of all divers, thou hast dived the deepest. That head upon which the upper sun now gleams, has moved amid this world's foundations. Where unrecorded names and navies rust, and untold hopes and anchors rot; where in her murderous hold this frigate earth is ballasted with bones of millions of the drowned; there, in that awful water-land, there was thy most familiar home. Thou hast been where bell or diver never went; hast slept by many a sailor's side, where sleepless mothers would give their lives to lay them down. Thou saw'st the locked lovers when leaping from their flaming ship; heart to heart they sank beneath the exulting wave; true to each other, when heaven seemed false to them. Thou saw'st the murdered mate when tossed by pirates from the midnight deck; for hours he fell into the deeper midnight of the insatiate maw [...]. O head! Thou hast seen enough to split the planets and make an infidel of Abraham, and not one syllable is thine!" (Melville 1851a, 339-340)

Monologue tragique, vaine interrogation du mystère, questionnement, dirait Victor Hugo, de la Bouche d'ombre. Mais " unrecorded names ", " untold hopes », " not one syllable » : c'est bien, en marge de leur disparition visuelle, au langage qu'échappent ces corps en partance vers les obscurités du néant. À l'impossibilité de la 
représentation figurée se substitue donc la figuration de l'interdit du langage. Le mutisme du sphinx noir, transposition sonore des profondeurs obscures et inaccessibles où évolue le cachalot, se fait ainsi l'expression vocale de la vacuité, point paradoxal et extrême de la blancheur.

Il faut, pour mesurer la pleine valeur de cette transfiguration synesthésique, en revenir à la définition même que donne du blanc Vassily Kandinsky. Selon le peintre, le blanc est en effet

le symbole d'un monde d'où toutes les couleurs, en tant que propriétés matérielles et substances, auraient disparu. Ce monde est tellement au-dessus de nous qu'aucun son ne nous en parvient. Il en vient un grand silence qui nous apparaît, représenté matériellement, comme un mur froid à l'infini, infranchissable, indestructible. C'est pourquoi le blanc agit également sur notre âme (psyché) comme un grand silence, absolu pour nous. Il résonne intérieurement comme un non-son, ce qui correspond sensiblement à certains silences en musique. (Kandinsky 155)

"Non-son »: on retrouve, dans le silence de la blancheur, la négativité même qui définissait à nos yeux, tout à l'heure, la figuration sphinxiale. On ne laisse pas de reconnaître par ailleurs dans ce «mur» infranchissable, dressé à l'infini, l'obstacle commun d'Achab et d'Ismaël, Moby-Dick, «mur de prison » pour le capitaine (Melville 1851a, 178), "mur aveugle et mort » pour le narrateur (" dead blind wall », Melville 1851a, 368). C'est bien de fait la baleine blanche, «brute muette " ( dumb brute», Melville 1851a, 178) évoquant la "vacuité muette» ("dumb blankness", Melville 1851a, 212) des paysages enneigés, qui est figurée sous les traits de ce monument secret, concrétion de mutisme sphinxial, qu'est la tête décapitée du cachalot. Signe supplémentaire, s'il en était encore besoin, que le mutisme du sphinx noir n'est que l'équivalent vocal de la blancheur du cétacé, la transposition sonore de son absence visuelle'.

\section{Fonction herméneutique : le hiéroglyphe muet ou la "lettre blanche »}

Dessin obscur greffé sur le corps blanc de Moby-Dick, le sphinx ne laisse pas en définitive d'évoquer ces traits mystérieux qui couvrent la peau de la baleine et que le narrateur a tôt fait d'apparenter explicitement aux hiéroglyphes égyptiens. Marques profondes s'offrant au seul regard averti, «signes mystiques » par lesquels la baleine demeure à tout jamais "indéchiffrable $»^{10}$. L'identification n'est pas fortuite. Elle fait référence en effet, dans l'imaginaire américain, aux conceptions transcendantalistes de l'écriture hiéroglyphique ${ }^{11}$. Nourries à la source de l'Egyptian Revival ${ }^{12}$, ces dernières puisent dans toute la tradition du Liber mundi, qui fait du monde un livre hiéroglyphique, symbolisant et symbolisable, offert à l'écriture et à l'interprétation de sages initiés. Or, comme nous l'avons montré ailleurs, la conception syncrétique d'un sphinx égypto-hellénique, détenteur des secrets de l'univers, interrogé par quelque CEdipe de passage, sert, depuis l'Antiquité, de figure emblématique à la Weltanschauung symboliste $^{13}$. Au-delà de la rémanence visuelle du colosse de Gizeh et du pouvoir pictural qui lui est associé, le choix de la figure du sphinx procède donc ici d'une intention fondamentalement herméneutique. Peindre la baleine blanche sous la forme d'un sphinx noir, c'est affirmer une volonté d'emprise tout à la fois métaphysique et langagière sur le réel. En montrer les limites, c'est esquisser la fracture fondamentale 
qui préside à toute signification, dessinant, derrière l'impossibilité de la représentation symbolique, l'éventualité d'un naufrage du sens.

Ismaël tente de fait, neuf chapitres après Achab, de décrypter la face du cachalot, pour s'en faire, dit-il explicitement, le Champollion. Ainsi au chapitre 79 s'essaie-t-il à une lecture physiognomonique de la tête noire décapitée. Mais l'animal demeure «sans langue " («tongueless », Melville 1851a, 380), muré dans «un silence pyramidal» ("pyramidical silence », Melville 1851a, 380), invitant Ismaël à sa poursuite comme " les empreintes dans la neige " attirent le chasseur. Heuristique de la trace noire sur fond blanc qui fait resurgir, naturellement dans le contexte égyptisant, l'image sphinxiale. Le cachalot n'offre au disciple de Lavater, comme à tout autre interprète, que «le vaste firmament du front sillonné d'énigmes, dont la mise à la mer est une muette menace de perdition pour les canots, les navires et les équipages " (Melville $1851 \mathrm{~b}, 384)^{14}$, souvenir quasi explicite de l'adresse tragique du capitaine Achab au monstre décapité. Et Ismaël de confirmer l'écho intratextuel en assurant que le cachalot est, d'un point de vue physiognomonique, «un Sphinx $»^{15}$. De l'un à l'autre, d'Achab à Ismaël, du chapitre 70 au chapitre 79, de The Sphynx à a Sphinx, presque rien : une voyelle, le "i grec " précisément, l'indéfinition d'un article, l'accès au corps du texte. Pas une rupture de la narration, ni même une fêlure, un repli tout au plus. Un repli tout de même. La vision mythique est entrée dans la langue, la comparaison est devenue antonomase, le dessin s'est fait mot. Échappant définitivement à toute capture symbolique pour devenir métaphore mobile, ce sphinx rendu au simple statut de nom commun consacre, à sa manière, l'échec de toute entreprise absolutiste et totalisante. Aux Ædipes de passage, qu'ils soient chasseur de baleine ou narrateur, le cachalot noir rappelle qu'il n'est de sphinx vivant que indéfini et insaisissable. Résolument blanc, donc.

Telle est donc la tête noire hissée sur le flanc du Pequod. Hiéroglyphe des hiéroglyphes, condensant les composantes figurative, symbolique et phonique de l'écriture égyptienne au lieu même du silence, pour se faire cryptogramme muet, signe visible du néant. Née du désir de donner forme à la baleine, elle rend tangible l'absence même de cette forme dans le langage. Elle s'apparente in fine à cette lettre $\mathrm{H}$, dit silent $\mathrm{H}$ en anglais, qui contient, selon la première page du roman, "presque à elle seule la signification » du vocable whale et sans qui la baleine, whale, ne serait que wale, rune obscure. Approximation de la signification littérale du cétacé, cette lettre signale d'emblée, noir sur blanc, que jamais l'objet du récit ne sera épuisé par les mots. Et le sphinx noir d'incarner ce que Claude Richard a nommé précisément «la lettre blanche » de la baleine (Richard 98), son absence signifiée.

\section{Fonction philosophique : entre espoir et néant, les portes de l'ombre}

Sphinx de l'emprise littéraire ou monstre de la quête halieutique, cachalot noir ou baleine blanche, qu'importe en définitive. L'issue est la même. Qu'il s'agisse de l'interrogation concrète du cachalot-sphinx par Achab ou de son appréhension rétrospective par Ismaël, dans les deux cas, le questionnement se fait vain, l'énigme demeure sans réponse, le sphinx paraît destiné à se taire. Point de confrontation réelle donc, fût-elle ironique, point de dialogue, fût-il agressif, point de solution, fût-elle énigmatique. Il y a là, à dire vrai, une constante plus particulière de l'imaginaire 
melvillien, qu'il convient d'interroger. Sa vie durant, en effet, l'auteur s'est heurté à un obstacle silencieux qu'il aime à représenter, à l'occasion, sous les traits de la figure mythique. Une de ses dernières œuvres, Clarel, long et obscur récit philosophique, en témoigne. Le sphinx, incessamment questionné, demeure, jusque dans l'épilogue, indifférent aux demandes des hommes :

Unmoved by all the claims our times avow,

The ancient Sphinx still keeps the porch of shade.

(Melville 1876, 498) cachalot, perçu dans la confrontation avec ses poursuivants, apparaît enfin dans toute sa mobilité et sa vivacité naturelles. Voilà qui invite pour finir à délaisser le portrait en noir et blanc de l'animal pour lui préférer les aventures hautes en couleur des 
baleiniers - américains, indiens, cambodgiens - hommes d'équipages à la complexion " jaune tigre ", second lieutenant "rouge de santé ", Queequeg enfin à la peau tannée, dont le corps recouvert de tatouages multicolores se confond avec la courtepointe bigarrée. Là, dans la polychromie du roman d'aventures, tandis que le grand sphinx muet laisse place à la loquacité de la sphinx hellénique, le crépusculaire «sphinx tête de mort » se pare des vivantes couleurs des papillons diurnes.

\section{BIBLIOGRAPHIE}

\section{Primaire}

Melville, Herman. Clarel (1876). The Writings of Herman Melville. Evanston and Chicago: The Newberry Library \& Northwestern UP, vol. 12, 1991.

Melville, Herman. Moby-Dick or, The Whale (1851). New York: Penguin Books, 1992, d'après l'édition de Northwestern UP, 1988 (indiqué Melville 1851a).

Melville, Herman. Moby-Dick. Texte traduit et présenté par Philippe Jaworski. Cuvres III. Paris : Gallimard, « Bibliothèque de la Pléiade », 2006 (indiqué Melville 1851b).

\section{Secondaire}

Bleikasten, André. « Moby Dick et la question du livre ». Revue française d'études américaines 50 (novembre 1991). Nancy : PU de Nancy, 377-390.

Blanchot, Maurice. « Le secret de Melville ». Faux-Pas. Paris : Gallimard, 1943, 27-277.

Derail-Imbert, Agnès. Moby-Dick. Allures du corps. Paris : Presses de l'ENS, 2000.

Irwin, John. American Hieroglyphics. The Symbol of the Egyptian Hieroglyphics in the American Renaissance. New Haven, Conn. \& London: Yale UP, 1980.

Jaworski, Philippe. Melville. Le désert et l'empire. Paris : Presses de l'ENS, 1986.

Jaworski, Philippe. "The Whiteness of the Whale, silence du chant ». Revue française d'études américaines 50 (novembre 1991), Nancy : PU de Nancy, 351-376.

Kandinsky, Vassily. Du spirituel dans l'art, et dans la peinture en particulier, traduit de l'allemand par Nicole Debrand et du russe par Bernadette du Crest. Paris: Denoël, 1989.

Metlitsky Finkelstein, Dorothee. Melville's Orienda. New Haven \& London: Yale UP, 1961.

Renker, Elisabeth. Strike through the Mask, Herman Melville and the scene of writing. Baltimore: Johns Hopkins UP, 1996.

Revol-Marzouk, Lise. L'Énigme aux origines. Le sphinx dans l'Antiquité égyptienne, grecque et romaine. Paris : Garnier, « perspectives comparatistes », 2016.

Revol-Marzouk, Lise. « Le sphinx, la peste et le choléra ou l'épidémie intérieure. Contamination et déplacements (Sophocle, Poe) ». La Contamination : lieux symboliques et espaces imaginaires. Paris : Classiques Garnier, 2012, 297-317. 
Revol-Marzouk, Lise. Le Sphinx et l'Abîme. Sphinx maritimes et énigmes romanesques dans Moby-Dick et Les Travailleurs de la mer. Grenoble : Ellug, 2008.

Revol-Marzouk, Lise. Le Sphinx, de l'Antiquité au Romantisme. Étude sur la constitution d'un mythe poétique, thèse de doctorat sous la direction de Pierre Brunel, université Paris-Sorbonne, 2003.

Richard, Claude. « Herman Melville. La lettre blanche de la baleine ». Lettres Américaines (1987), Alinéa, 75-112.

Richardson, R.D. Myth and Literature in the American Renaissance. Bloomington: Indiana UP, 1978.

Vincent, Howard. The Trying-Out of Moby-Dick. Boston : Houghton Mifflin, 1948.

\section{NOTES}

1. On désigne couramment en français le monstre hellénique par l'article féminin (" la sphinx ») pour le distinguer de son mâle homonyme égyptien («le sphinx »). Notre langue propose par ailleurs, depuis 1589, l'usage pour la figure grecque du substantif féminin "sphinge ", issu également du latin sphinx, sphingis. Nous avons choisi toutefois, dans le présent essai, de n'utiliser que la forme "sphinx ", accompagnée au besoin de l'un ou l'autre article, afin de mieux rendre compte de la rencontre possible entre sphinx grecs et égyptiens dans l'imaginaire de Melville.

2. Il s'agit là d'une condensation mythique récurrente au XIX ${ }^{\mathrm{e}}$ siècle entre les deux types principaux de sphinx antiques : le lion couchant à tête de pharaon, statue du souverain divinisé après sa mort, en Égypte d'une part; la lionne ailée à buste et tête de femme, monstre poseur d'énigmes vaincu par CEdipe d'autre part. La convergence entre ces deux figures puise dans une tradition qui remonte à l'Antiquité, et dont nous avons étudié le développement dans la littérature et l'art occidentaux des origines au Romantisme : voir Lise Revol-Marzouk, Le Sphinx, de l'Antiquité au Romantisme. Étude sur la constitution d'un mythe poétique, thèse de doctorat sous la direction de Pierre Brunel, Paris: Université Paris-Sorbonne, 2003. Pour une étude plus spécifique sur les sphinx antiques, voir également Lise Revol-Marzouk, L'Énigme aux origines. Le sphinx dans l'Antiquité égyptienne, grecque et romaine, Paris: Garnier, «perspectives comparatistes ", 2016.

3. Nous empruntons l'expression à R.D. Richardson qui analyse en ces termes la manière dont Moby-Dick figure la rencontre entre réel et imaginaire, à la source du processus de création mythique (R.D. Richardson, Myth and Literature in the American Renaissance, Bloomington : Indiana UP, 1978, Chapitre 7 « Melville », § 3 « Mythic investiture in Moby-Dick», 210-226). L'investiture mythique constitue dans cette optique une technique fictionnelle par laquelle l'invisible est incarné dans le visible.

4. L'illustration du face-à-face légendaire procède en effet à l'époque classique de l'opposition entre l'immobilisme symétrique du monstre et le dynamisme asymétrique d'œdipe, appuyé sur un bâton ou sur sa lance.

5. Sis au pied d'un plateau rocheux, dans une dépression géologique, le grand sphinx est en effet régulièrement recouvert jusqu'au cou par les sables. Amorcé en 1816 sous la direction de l'explorateur génois Giovanni Caviglia, son désensablement permet d'en exhumer dès 1818 les pattes, l'uraeus, la barbe et une stèle posée entre les pattes. Le sable ayant de nouveau recouvert partiellement la statue, ne laissant apercevoir que sa tête, l'égyptologue et philologue allemand Karl Richard Lepsius se voit contraint, à peine vingt-cinq ans plus tard, de désensabler une nouvelle fois la stèle et la chapelle dont elle constitue le fond. À la suite de Lepsius, c'est enfin l'égyptologue français Auguste Mariette qui s'attaque en 1853 au désensablement du grand Sphinx, dont il dégage tout le temenos. En 1858, au cœur même d'un siècle d'Egyptian revival en Europe comme aux États-Unis, le grand Sphinx de Gizeh est, pour la première fois, libéré des 
déblais. Mais artistes et écrivains occidentaux demeurent longtemps sensibles au souvenir de cette tête humaine immense semblant flotter sur les sables du désert.

6. "In fact, as the great Hunter says, the mere skeleton of the whale bears the same relation to the fully invested and padded animal as the insect does to the chrysalis that so roundingly envelopes it. This peculiarity is strikingly evinced in the head, as in some part of this book will be incidentally shown » (Melville 1851, 289).

7. On peut en effet identifier chez Melville une référence cachée à ce lépidoptère, dont le thorax noir porte une figure blanche en forme de tête de mort, qui lui vaut son nom de «sphinx-tête-demort ». L'usage n'en est pas rare dans un tel contexte herméneutique chez les auteurs américains du XIX siècle. Nous l'avons étudié par exemple dans le conte «The Sphinx » d'Edgar Allan Poe, réécriture du mythe d'ÆEdipe plaçant, sous l'égide d'un papillon "sphinx » aux traits égyptohelléniques, une réflexion sur la représentation et ses énigmes. Herman Melville connaît d'ailleurs probablement ce conte publié pour la première fois en 1846 dans le Arthur's Ladies' Magazine. Voir L. Revol-Marzouk, "Le sphinx, la peste et le choléra ou l'épidémie intérieure. Contamination et déplacements (Sophocle, Poe) », La Contamination: lieux symboliques et espaces imaginaires, Paris : Classiques Garnier, 2012, 297-317.

8. L'expression désigne explicitement la blancheur, à la fin du chapitre 42 (Melville 1851, 212).

9. Sur cette fonction phonique de la blancheur, voir l'article de Philippe Jaworski « The whiteness of the whale, silence du chant » (Revue française d'études américaines 50 (1991) : 351-375), qui, sans évoquer explicitement la figure du sphinx, ne laisse pas cependant de nourrir notre réflexion.

10. Ces marques sont évoquées, avec leur analogie hiéroglyphique, au chapitre 68 , "The Blanket » : "These are hieroglyphical; that is, if you call those mysterious ciphers on the walls of pyramids hieroglyphics, then that is the proper use word to use in the present connexion. [...] Like those mystic rocks, too, the mystic-marked whale remains undecipherable » (Melville 1851, 333).

11. Sur ce point, voir John Irwin, American Hieroglyphics. The Symbol of the Egyptian Hieroglyphics in the American Renaissance, New Haven, Conn. \& London : Yale UP, cop. 1980.

12. Sur l'orientalisme de Melville en général, et sur son accès au matériau égyptien en particulier, voir Dorothee Metlitsky Finkelstein, Melville's Orienda, New Haven \& London : Yale UP, 1961, 1-24 et 121-144 notamment.

13. Pour une monographie diachronique sur le sujet, voir Lise Revol-Marzouk, Le Sphinx, de l'Antiquité au Romantisme. Étude sur la constitution d'un mythe poétique, thèse de doctorat sous la direction de Pierre Brunel, Paris : Université Paris-Sorbonne, 2003. On en trouvera une synthèse dans l'introduction à Lise Revol-Marzouk, Le Sphinx et l'Abîme. Sphinx maritimes et énigmes romanesques dans Moby-Dick et Les Travailleurs de la mer, Grenoble: Ellug, "Ateliers de l'Imaginaire ", 2008, "Repères », 9-32. Pour une étude plus spécifique sur les sphinx antiques, voir également Lise Revol-Marzouk, L'Énigme aux origines. Le sphinx dans l'Antiquité égyptienne, grecque et romaine, Paris : Garnier, « perspectives comparatistes », 2016.

14. " [...] nothing but that one broad firmament of a forehead, pleated with riddles; dumbly lowering with the doom of boats, and ships, and men » (Melville 1851, 379).

15. «If the Sperm Whale be physiognomically a Sphinx [...]» (Melville 1851, 381).

16. Pour un approfondissement des perspectives sur le roman de Melville exposées dans cet article, en comparaison notamment avec l'expression du sphinx dans Les Travailleurs de la mer de Victor Hugo, autre roman maritime contemporain de Moby-Dick, voir Lise Revol-Marzouk, Le Sphinx et l'Abîme. Sphinx maritimes et énigmes romanesques dans Moby-Dick et Les Travailleurs de la mer, Grenoble : Ellug, « Ateliers de l'Imaginaire », 2008. 


\section{RÉSUMÉS}

Au cœur de Moby-Dick apparaît, hissée sur le flanc du Pequod, la tête noire d'un cachalot décapité. C'est là, au chapitre 70, que le capitaine Achab vient l'interroger, dans une mise en scène aux accents tragiques, sur les secrets des abîmes. Intitulé "The Sphynx", l'épisode mêle habilement des éléments empruntés à la double origine culturelle de la figure mythique, réminiscences conjointes de la statue colossale de Gizeh et de la confrontation célèbre entre cEdipe et le monstre thébain. Ismaël avait promis au lecteur une "peinture" de la baleine blanche: le surgissement du sphinx, avec son réseau identifiable de traits référentiels, iconographiques et légendaires, participe assurément de cette tentative de capture métaphorique de l'ineffable animal. L'image s'avère pourtant décevante. Inlassablement interrogé, le monstre demeure sans voix. Simple profil d'ombre sur la page blanche du texte et de la baleine, le sphinx noir, loin de compenser le mutisme des signes, ne fait qu'exhiber, pour ainsi dire en négatif, l'énigme insoluble de la représentation.

At the heart of Moby-Dick appears the black decapitated head of a whale, hoisted against the Pequod's side. It is in this tragic passage in chapter 70 that Captain Ahab questions the head about the secrets of the abyss. The scene, entitled The Sphynx, cleverly introduces elements that draw on the dual origins of the mythical figure, the colossal statue at Giza and the famous confrontation between Oedipus and the Theban beast. Ishmael had promised to paint a "picture" of the white whale for the reader: the sudden appearance of the sphinx, together with its identifiable network of legendary, iconographic and referential features, is surely part of this attempt to metaphorically catch the indescribable animal. However, this picture proves to be a disappointment. The monster remains mute in the face of this relentless questioning. Far from compensating for the silence of the symbols, the black sphinx, casting its shadow on the white page of both the text and the whale, merely projects, as if in a photographic negative, the unsolvable enigma of representation.

\section{INDEX}

Mots-clés : sphinx, mythe, hiéroglyphe, herméneutique, représentation, vacuité, silence, noir, blancheur, ineffable

oeuvrecitee Moby-Dick

Keywords : sphinx, myth, hieroglyphics, hermeneutics, representation, blankness, silence, black, whiteness, indescribable

\section{AUTEURS}

\section{LISE REVOL-MARZOUK}

Lise Revol-Marzouk, ancienne élève de l'ENS Ulm et enseignante des Universités Paris 4 et Stanford, Maître de Conférences en Littérature Comparée à l'Université Grenoble 3, Membre du Centre de Recherche sur l'Imaginaire (Université Grenoble 3) et du Centre de Recherche en Littérature Comparée (Université Paris 4), spécialiste de mythocritique et mythopoétique. Auteur notamment de nombreux articles, d'une thèse et de plusieurs ouvrages sur les sphinx (Le Sphinx, de l'Antiquité au Romantisme. Étude sur la constitution d'un mythe poétique, Lille : ANRT, 2003 ; Le Sphinx et l'Abîme. Sphinx maritimes et énigmes romanesques dans Moby-Dick et Les Travailleurs de la 
mer, Grenoble : Ellug, coll. « Ateliers de l'Imaginaire », 2008 ; L'Énigme aux origines. Le sphinx dans l'Antiquité égyptienne, grecque et romaine, Paris : Garnier, coll. «Perspectives comparatistes », 2015). Ses travaux portent également sur la représentation et la relation entre textuel et visuel, en particulier sur les questions de l'énigme et du silence, du voyage et de l'aventure, du corps et de la contamination (La Contamination : lieux symboliques et espaces imaginaires, co-dir., Paris :

Classiques Garnier, 2012). 